Research Article

\title{
Identification of Key Genes and Pathways Associated with Sex Differences in Osteoarthritis Based on Bioinformatics Analysis
}

\author{
Shiying Wang, ${ }^{1,2}$ Huanmei Wang, ${ }^{3}$ Wei Liu $\mathbb{D}^{4},{ }^{4}$ and Biaofang Wei $\mathbb{D}^{2}$ \\ ${ }^{1}$ Guangzhou University of Chinese Medicine, Guangzhou, Guangdong Province 510006, China \\ ${ }^{2}$ Department of Femoral Head, Linyi People's Hospital, Linyi, Shandong Province 276000, China \\ ${ }^{3}$ Department of Rehabilitation, The Ninth Hospital of Wuhan, Wuhan, Hubei Province 430000, China \\ ${ }^{4}$ Department of Encephalopathy, The Second Affiliated Hospital of Shandong University of Traditional Chinese Medicine, Jinan, \\ Shandong 250001, China
}

Correspondence should be addressed to Biaofang Wei; biaofangwei@aliyun.com

Received 29 July 2019; Revised 11 November 2019; Accepted 13 November 2019; Published 6 December 2019

Academic Editor: Swaran J. S. Flora

Copyright (C) 2019 Shiying Wang et al. This is an open access article distributed under the Creative Commons Attribution License, which permits unrestricted use, distribution, and reproduction in any medium, provided the original work is properly cited.

Sex differences have been suggested to play critical roles in the pathophysiology of osteoarthritis (OA), resulting in sex-specific prevalence and incidence. However, their roles in the development of OA remain largely unknown. The aim of this study was to screen out key genes and pathways mediating biological differences between OA females after menopause and OA males. First, the gene expression data of GSE36700 and GSE55457 were downloaded from the Gene Expression Omnibus database. Differentially expressed genes (DEGs) between sexes were identified using R software, respectively. The overlapping DEGs were obtained. Then, protein-protein interactive (PPI) network was constructed to further analyze interactions between the overlapping DEGs. Finally, enrichment analyses were separately performed using Gene Ontology and Kyoto Encyclopedia of Genes and Genomes tools. In our results, a total of 278 overlapping DEGs were identified between OA females after menopause and OA males, including 219 upregulated and 59 downregulated genes. In the PPI network, seven hub genes were identified, including EGF, ERBB2, CDC42, PIK3R2, LCK, CBL, and STAT1. Functional enrichment analysis revealed that these genes were mainly enriched in PI3K-Akt signaling pathway, osteoclast differentiation, and focal adhesion. In conclusion, the results in the current study suggest that pathways of PI3K-Akt, osteoclast differentiation, and focal adhesion may play important roles in the development of OA females after menopause. EGFR, ERBB2, CDC42, and STAT1 may be key genes related to OA progression in postmenopausal women and may be promising therapeutic targets for OA.

\section{Introduction}

Osteoarthritis (OA), the most common musculoskeletal disorder, leads to functional disability and loss in quality of life. It affects women after menopause 2-3 times often than men [1]; worldwide disease estimates show that approximately $18 \%$ of women and $6 \%$ of men at the age of 60 years or older suffer from OA [2]. It is characterized by cartilage degradation, synovial inflammation, subchondral bone sclerosis, and chronic pain $[3,4]$. Unfortunately, adequate therapies cannot block or reverse OA progression apart from pain relief [5]. Eventually, joint replacement surgery is commonly applied to patients with advanced stage. It is therefore urgent to explore potential biomarkers and therapeutic targets for OA.

Sex differences in its prevalence suggest a significant role for sex hormones in the pathogenesis of OA. This has led to the hypothesis that female sex hormones may have protective effects on the risk of developing OA. A number of studies have assessed the association between sex hormone levels and the risk of OA, but with conflicting results. One observational study showed that decreased sex hormone levels were associated with an increased prevalence of hand $\mathrm{OA}$ in nonelderly females [6]. Spector et al. [7] reported that middle-aged women with $\mathrm{OA}$ had lower circulating sex hormone levels compared with healthy women. By contrast, 
a cohort study showed greater log-transformed concentrations of sex hormones were associated with a higher incidence of hip replacement for OA [8]. Overall, previous evidence for the sex-specific mechanism in $\mathrm{OA}$ is inconclusive.

Recently, microarray technology based on highthroughput platforms has been widely used to profile gene expression. A lot of effort has been spent in identifying or assessing specific genes and pathways for the progression of $\mathrm{OA}$. The majority of studies are focused on gene expression data in OA patients compared to healthy subjects [9-13]. But there is less information regarding the differences of gene expression data between sexes. Thus, the purpose of this study was to identify key genes and pathways contributing to biological differences between sexes by a comprehensive bioinformatics analysis. This study may improve the understanding of the sex-specific mechanism in $\mathrm{OA}$ and suggest potential biomarkers and therapeutic targets for OA treatment.

\section{Materials and Methods}

2.1. Microarray Data. In this study, we searched datasets from the Gene Expression Omnibus (GEO) repository (https://www.ncbi.nlm.nih.gov/geo/) with the keywords: "osteoarthritis" [MeSH Terms] OR “osteoarthritis" [All Fields] AND "Homo sapiens" [porgn] AND "gse"[Filter]. The screening standards were as follows: the microarray datasets were gene expression profiles; the clinical information in datasets contains gender and age. Eventually, two datasets were screened out for further analysis: GSE36700 [14] and GSE55457 [15]. Both datasets include synovial membrane samples from OA females and OA males. GSE36700 is based on GPL570 [HG-U133_Plus_2] Affymetrix Human Genome U133 Plus 2.0 Array (Affymetrix UK Ltd., High Wycombe, UK). GSE55457 is based on GPL96 [HG-U133A] Affymetrix Human Genome U133A Array (Affymetrix, Santa Clara, California, USA). According to our aims, only OA females after menopause and OA males in both datasets were selected for further analysis in the present study.

2.2. Differential Expression Analysis. We used software R (version 3.5.1) and related packages to compare differentially expressed genes (DEGs) between OA females after menopause and OA males in GSE36700 and GSE55457, respectively. Both datasets were first background adjusted and normalized by $\log _{2}$ transformation. Background correction, quantile normalization, and probe summarization of the raw microarray data were performed by robust multiarray average (RMA) algorithm [16] in Linear Models for Microarray Data (limma) package [17]. The detailed analysis was according to the manufacturer's protocol. Then, $P$ values were adjusted for comparisons using the false discovery rate (FDR) of the Benjamini and Hochberg $(\mathrm{BH})$ test [18] in limma package [17]. DEGs were selected with the commonly used thresholds of a $P$ value $<0.05$ and $\mid \log _{2}$ fold change (FC) $\mid>0.5[19,20]$. Volcano plot of DEGs was generated by ggplot2 package in $\mathrm{R}$ software. Furthermore, the overlapping DEGs were the intersection of DEGs in two datasets.

2.3. Functional Enrichment Analysis. The Database for Annotation, Visualization, and Integrated Discovery (DAVID, version 6.8, https://david.ncifcrf.gov/) [21] is a wellknown online tool that provides comprehensive information for list of DEGs by Gene Ontology (GO) and the Kyoto Encyclopedia of Genes and Genomes (KEGG) pathway analyses. In order to obtain the biological function and signaling pathways of DEGs, we undertook GO enrichment and KEGG enrichment analyses for DEGs through DAVID. Only enriched gene count of GO and KEGG terms more than or equal to 3 and a $P$ value $<0.05$ were considered as statistically significant.

2.4. Protein-Protein Interaction (PPI) Network Analysis. In order to identify functional interactions between the products of the overlapping DEGs, the overlapping DEGs were uploaded to the Search Tool for the Retrieval of Interacting Genes (STRING, version 11.0, https://www. string-db.org/) [22], which is an open accessible online database of genetic and protein interactions. The PPI network was constructed via STRING with the default threshold of a combined score $>0.4$. Then, we performed Cytoscape software (version 3.7.2) [23] to visualize the PPI network. In addition, nodes in the PPI network represent proteins, while the edges represent the interactions. The hub genes were those highly connected proteins possessing important biological function with a node degree greater than or equal to 10 .

\section{Results}

3.1. Identification of Overlapping DEGs. A total of 3395 and 1387 DEGs were identified in synovial membranes from OA females after menopause compared with OA males in GSE36700 and GSE55457, respectively. Among them, 2077 upregulated and 1318 downregulated genes were obtained from GSE36700; meanwhile, 1020 upregulated and 367 downregulated genes were obtained from GSE55457. The volcano plots are shown in Figure 1, in which the red represents upregulated genes and the green represents downregulated genes. Furthermore, a total of 278 overlapping genes were identified in two datasets after the intersection, including 219 upregulated and 59 downregulated genes. As shown in Figure 2, the Venn diagrams displayed the overlapping genes in two datasets after the intersection.

3.2. GO Enrichment Analysis. The GO enrichment analysis was performed to determine the biological function of the overlapping DEGs. A total of 50 GO terms are remarkably enriched for the overlapping DEGs. The overlapping DEGs were mainly enriched in positive regulation of transcription from RNA polymerase II promoter, signal transduction, cell adhesion, positive regulation of transcription, 

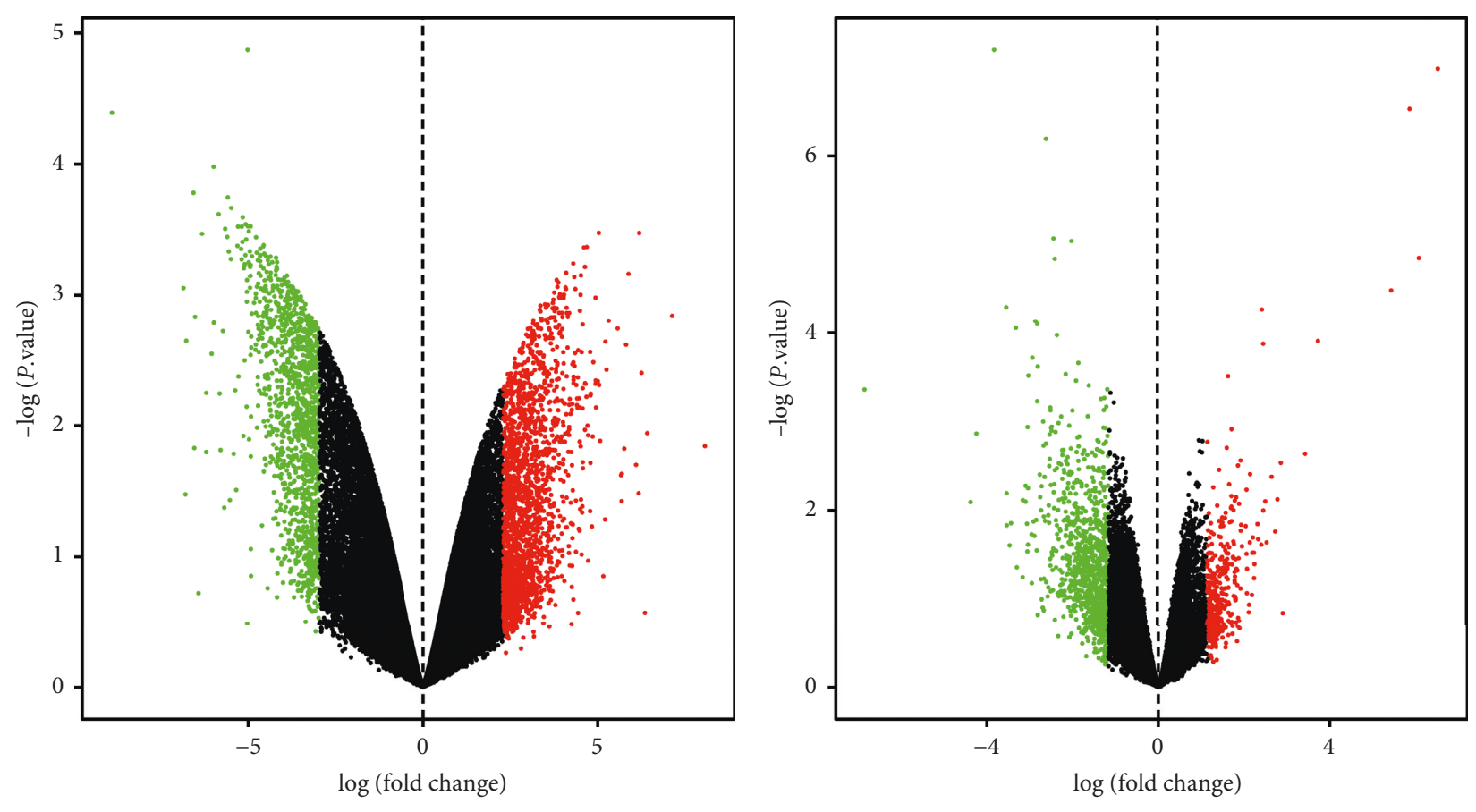

$$
\begin{aligned}
& \text { Category } \\
& \text { - Down } \\
& \text { - Normal } \\
& \text { - Up }
\end{aligned}
$$

$$
\begin{aligned}
& \text { Category } \\
& \text { - Down } \\
& \text { - Normal } \\
& \text { - Up }
\end{aligned}
$$

(a)

(b)

FIGURe 1: Volcano plots of DEGs in OA females after menopause versus OA males in (a) GSE36700 and (b) GSE55457.

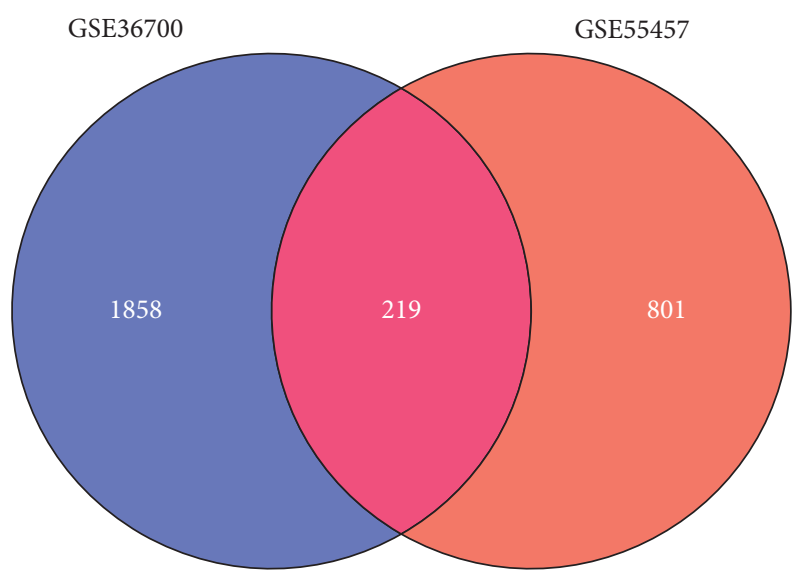

(a)

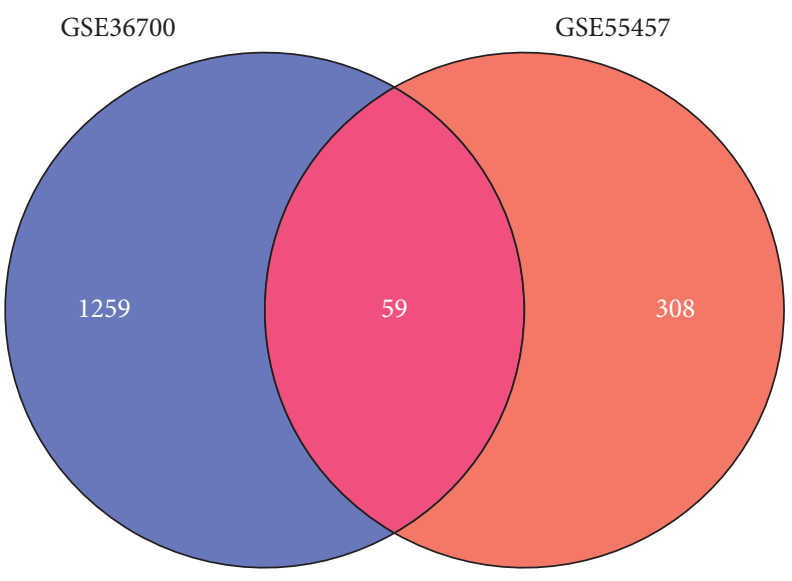

(b)

FIGURE 2: Venn diagrams of (a) upregulated and (b) downregulated overlapping DEGs in GSE36700 and GSE55457.

DNA-templated, protein phosphorylation, positive regulation of GTPase activity, positive regulation of cell proliferation, and regulation of phosphatidylinositol 3-kinase signaling. The top 20 GO terms are shown in Figure 3 and Table 1.

3.3. KEGG Pathways Analysis. KEGG pathways analysis was performed to explore the enriched pathways of the overlapping DEGs. As shown in Figure 4, a total of 15 pathways are identified. The pathways related to sex differences in OA are mainly enriched as follows: PI3KAkt signaling pathway ( $P$ value $=4.59 E-02$, which involved epidermal growth factor (EGF) and phosphoinositide-3-kinase regulatory subunit 2 (PIK3R2)), osteoclast differentiation $(P$ value $=1.23 E-02$, which involved PIK3R2 and signal transducer and activator of transcription 1 (STAT1)), and focal adhesion $(P$ value $=3.10 E-02$, which involved EGF, PIK3R2, and erbb2 receptor tyrosine kinase 2 (ERBB2)). 


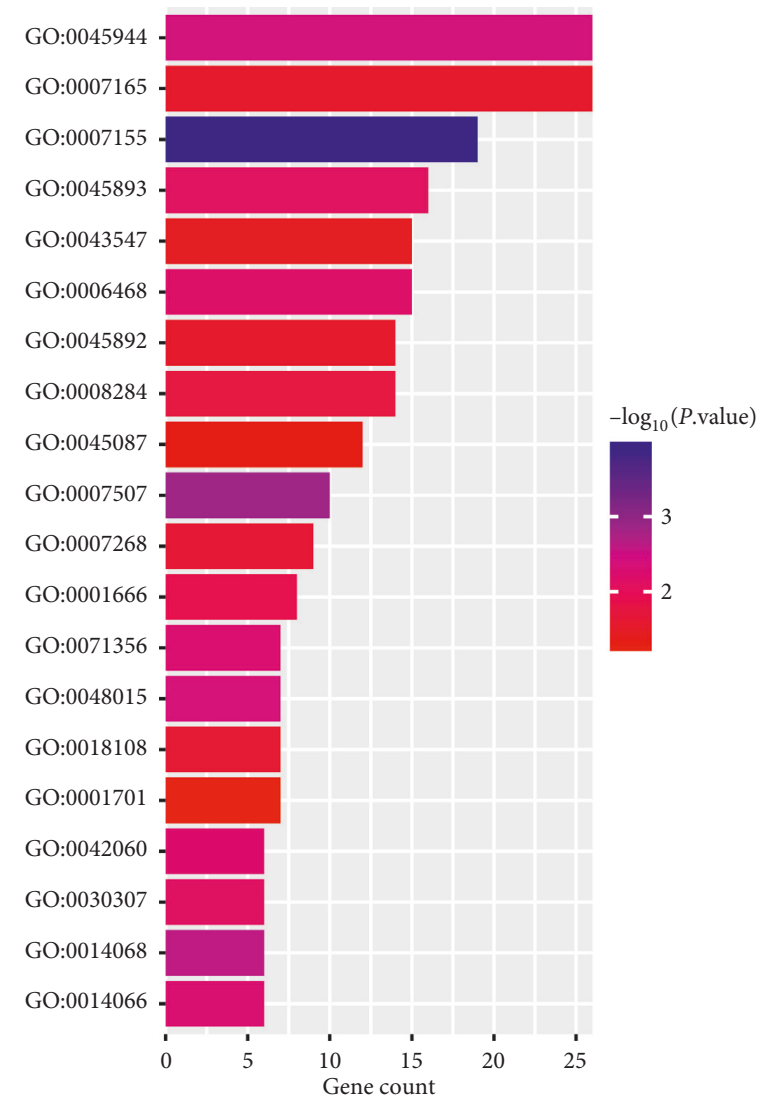

Figure 3: The top $20 \mathrm{GO}$ enrichment analysis of the overlapping DEGs of OA females after menopause versus OA males.

TABle 1: The top 20 GO enrichment analysis of the overlapping DEGs associated with sex in OA.

\begin{tabular}{|c|c|c|c|}
\hline GO term & Biological function & Gene count & $P$ value \\
\hline GO:0045944 & $\begin{array}{l}\text { Positive regulation of transcription from RNA } \\
\text { polymerase II promoter }\end{array}$ & 26 & $3.86 E-03$ \\
\hline GO:0007165 & Signal transduction & 26 & $2.76 E-02$ \\
\hline GO:0007155 & Cell adhesion & 19 & $1.18 E-04$ \\
\hline GO:0045893 & Positive regulation of transcription, DNA-templated & 16 & $7.62 E-03$ \\
\hline GO:0006468 & Protein phosphorylation & 15 & $6.31 E-03$ \\
\hline GO:0043547 & Positive regulation of GTPase activity & 15 & $3.39 E-02$ \\
\hline GO:0008284 & Positive regulation of cell proliferation & 14 & $1.75 E-02$ \\
\hline GO:0045892 & Negative regulation of transcription, DNA-templated & 14 & $2.80 E-02$ \\
\hline GO:0045087 & Innate immune response & 12 & $4.65 E-02$ \\
\hline GO:0007507 & Heart development & 10 & $1.33 E-03$ \\
\hline GO:0007268 & Chemical synaptic transmission & 9 & $2.28 E-02$ \\
\hline GO:0001666 & Response to hypoxia & 8 & $1.22 E-02$ \\
\hline GO:0048015 & Phosphatidylinositol-mediated signaling & 7 & $4.25 E-03$ \\
\hline GO:0071356 & Cellular response to tumor necrosis factor & 7 & $5.09 E-03$ \\
\hline GO:0018108 & Peptidyl-tyrosine phosphorylation & 7 & $2.32 E-02$ \\
\hline GO:0001701 & In utero embryonic development & 7 & $5.29 E-02$ \\
\hline GO:0014068 & $\begin{array}{c}\text { Positive regulation of phosphatidylinositol 3-kinase } \\
\text { signaling }\end{array}$ & 6 & $2.40 E-03$ \\
\hline GO:0014066 & Regulation of phosphatidylinositol 3-kinase signaling & 6 & $5.27 E-03$ \\
\hline GO:0042060 & Wound healing & 6 & $5.86 E-03$ \\
\hline GO:0030307 & Positive regulation of cell growth & 6 & $7.19 E-03$ \\
\hline
\end{tabular}

3.4. PPI Network Construction. To aid in the understanding of the interactions between the overlapping DEGs, PPI network was constructed using the STRING database. The
PPI network was composed of 243 nodes (the overlapping DEGs) and 330 edges (interactions between the overlapping DEGs). The genes with higher scores were the hub genes, as 


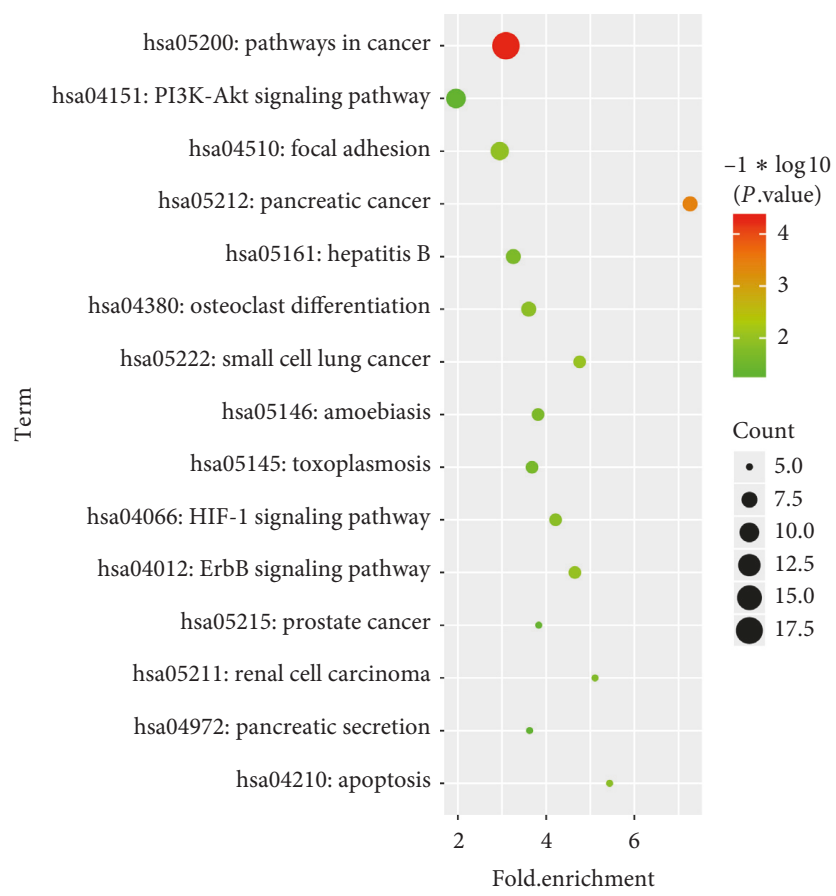

FIGURE 4: Enriched pathways of the overlapping DEGs in OA females after menopause and OA men.

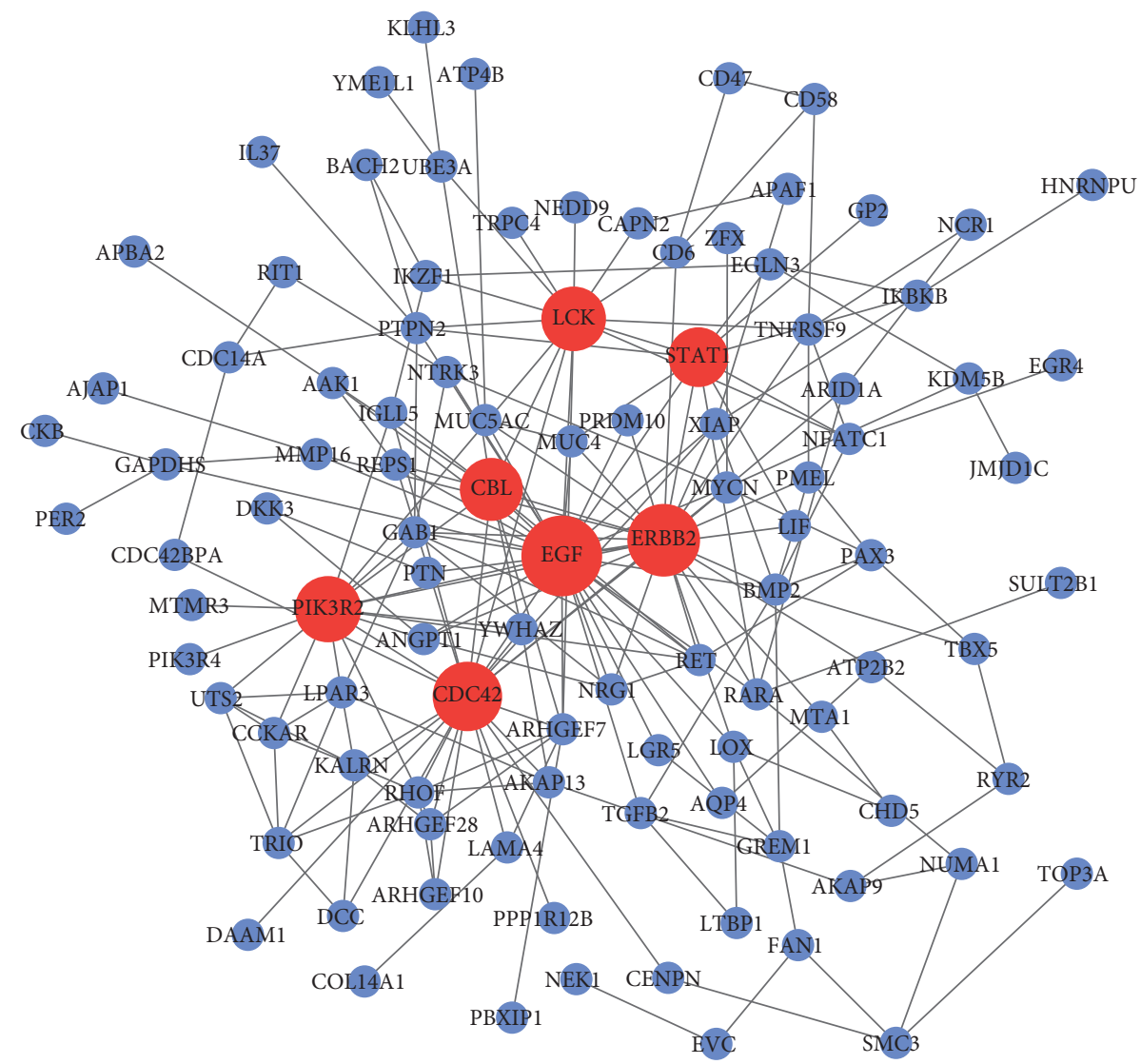

Figure 5: PPI network of the overlapping DEGs in OA females after menopause and OA men.

the genes of higher degree may be associated with OA. As displayed in Figure 5, the hub genes with node degree greater than or equal to 10 are EGF (degree=30), ERBB2
$($ degree $=25)$, cell division cycle $42($ CDC42 $)($ degree $=24)$, PIK3R2 (degree $=17$ ), LCK protooncogene, Src family tyrosine kinase $(\mathrm{LCK})($ degree $=15)$, Cbl protooncogene $\mathrm{B}$ 
$(\mathrm{CBL}) \quad($ degree $=12)$, and STAT1 (degree=11). Among them, EGF, ERBB2, PI3KR2, and LCK were upregulated in $\mathrm{OA}$ postmenopausal women compared to OA men; meanwhile, CDC42, CBL, and STAT1 were downregulated in OA postmenopausal women compared to OA men.

\section{Discussion}

$\mathrm{OA}$ is a degenerative joint disease with sex-specific prevalence and incidence. The prevalence of $\mathrm{OA}$ is higher among women than among men, and the risk for developing OA increases among women after menopause [24]. Sex hormones help in explaining sex differences in OA, albeit partly. After menopause, the level of estrogen is reduced continuously while the incidence of $\mathrm{OA}$ is rising dramatically, suggesting estrogen may have a protective effect on OA. Unfortunately, long-term use of estrogen cannot block or reverse OA progression but increases the risk of cancers such as breast cancer and endometrial cancer $[25,26]$. The underlying mechanisms contributing to sex differences in $\mathrm{OA}$ are still poorly understood. It is important to study the molecular mechanisms of sex differences in OA.

To our knowledge, our study, for the first time, identified key genes and pathways in synovial membrane of OA females after menopause compared to OA males using bioinformatics analysis. In the present study, we performed an integrated bioinformatics analysis using two GEO datasets of gene expression profiles to identify the biological mechanisms involved in the pathogenesis of sexes differences in OA. A total of 278 overlapping DEGs were identified between OA postmenopausal women and OA men, including 219 upregulated and 59 downregulated ones. Among the overlapping DEGs, EGF, ERBB2, CDC42, PIK3R2, LCK, $\mathrm{CBL}$, and STAT1 were identified as the hub genes. GO enrichment analysis of the overlapping DEGs showed that the hub genes EGF, ERBB2, and PIK3R2 were mainly enriched in regulation of phosphatidylinositol 3-kinase (PI3K) signaling, positive regulation of GTPase activity, and phosphatidylinositol-mediated signaling. Besides, KEGG pathways of the overlapping DEGs revealed that these hub genes were mainly enriched in pathways of PI3K-Akt, focal adhesion, and osteoclast differentiation. The results of the present study were reasonable and consistent with the results of previous studies, which have also suggested that PI3K-Akt signaling pathway [27-29], focal adhesion [30], and osteoclast differentiation $[31,32]$ are involved in the pathogenesis of $\mathrm{OA}$.

In the PPI network construction, we screened out seven hub genes EGF, ERBB2, CDC42, PIK3R2, LCK, CBL, and STAT1. All these genes may play important roles in the occurrence and progression in $\mathrm{OA}$, particularly in $\mathrm{OA}$ females after menopause. Mounting evidence highlights an important role of EGF in the pathogenesis of OA. EGF is an active polypeptide composed of 53 amino acids. Epidermal growth factor receptor (EGFR, ERBB1) is a member of the receptor tyrosine kinases (RTKs) family which also includes ERBB2/HER2, ERBB3/HER3, and ERBB4/HER4. EGFR, with intrinsic tyrosine kinase activity, can be bound and activated by a family of several peptide growth factors including EGF [33]. Compared with EGFR, ERBB2 does not contain a ligand-binding domain. The activation of EGFR occurs through trans-autophosphorylation of the intracellular kinase domain. Phosphorylated EGFR initiates myriad of key signaling pathways, among which the most studied are PI3K/Akt, JAK/STAT, and so on. In addition, PI3K/Akt acts as one of the vital signaling pathways in the development of OA [34]. In non-small-cell lung cancer, high expression of EGFR correlates with poor survival and anti-EGFR agents have greatly improved the progression-free survival over standard chemotherapy [35]. In breast cancer, overexpression of HER2 is generally associated with poor prognosis and HER2 targeted therapies have significantly improved the therapeutic outcome [36]. In the late stage of OA, human OA samples contain significantly more activated EGFR than controls; gefitinib could efficiently inhibit EGFR functions in OA joints and restore cartilage structure and function in the mouse model [37]. As to ERBB2, it is highly expressed in rheumatoid arthritis synovial cells compared to controls [38]. Similarly, CDC42, a member of Rho family small GTPases, is highly expressed in both articular cartilage and subchondral bone in a mouse OA model; knockdown of Cdc42 expression or inhibition of Cdc42 activity robustly attenuates knee joint destruction in mouse [39]. STAT1 has been widely regarded as a significant transcription factor involved in joint inflammation and destruction [40]. In addition to the genes that have already been discussed, there is a lack of data regarding whether $\mathrm{PI} 3 \mathrm{KR} 2, \mathrm{LCK}$, and $\mathrm{CBL}$ have strong connections with $\mathrm{OA}$ occurrence and progression. Given the specific pathophysiologic roles of EGFR, ERBB2, CDC42, and STAT1 in the development of joints, and investigation into targeted inhibition of these receptors in the setting of $\mathrm{OA}$ is of great interest.

The pathogenesis of $\mathrm{OA}$ is multifactorial and intricate and is characterized by cartilage destruction, synovial inflammation, and subchondral bone sclerosis. It is widely accepted that the PI3K/Akt signaling pathway plays a critical role in regulating diverse cellular processes including cell proliferation, apoptosis, metabolism, differentiation, and cell cycle [41]. Activation of PI3K signal transduction pathway has been recently recognized to be one of the important mechanisms for antiapoptosis of cells. Researchers found that activation of PI3K-Akt signaling pathway can increase matrix metalloproteinase (MMP) production which results in the progression of OA, while inhibition of PI3K-Akt signaling pathway attenuates the development of OA [34]. Further researches indicated that inhibition of PI3K-Akt signaling pathway attenuates inflammatory response in rats with OA [42]. In the current study, the upregulated gene PI3KR2 was enriched in PI3K-Akt signaling pathway in OA postmenopausal women. Prompted by the overexpressed PI3KR2 in cancers which correlates with PI3K activation [43], we could assume that the upregulated PI3KR2 might be related to the activation of PI3K-Akt signaling pathway in OA. Subsequently, the activated PI3K-Akt pathway contributes to the occurrence and development of OA. This may partly explain why OA occurs more frequently in postmenopausal women than in men. Yet, this is still required to 
be further confirmed by additional biochemistry and clinicrelated studies.

The KEGG pathways of the overlapping DEGs revealed that the hub genes were mainly enriched in osteoclast differentiation and focal adhesion as well. It is now well established that in OA, the balance between osteoclast-induced bone resorption and osteoblast-induced remodeling is being progressively deregulated [44]. Osteoclasts are multinucleated cells derived from hematopoietic stem cells of monocytic line and specialized for bone resorption. Studies have found increased bone resorption in patients with progressive OA compared to nonprogressive OA [45]. Our findings for these enrichments in OA suggest that osteoclast differentiation and focal adhesion may play an important part in the development of OA, especially in postmenopausal patients.

In summary, the results in the current study suggest that pathways of PI3K-Akt, osteoclast differentiation, and focal adhesion may play important roles in the development of OA females after menopause. EGFR, ERBB2, CDC42, and STAT1 may be key genes related to OA progression in postmenopausal women and may be promising therapeutic targets for OA.

\section{Data Availability}

The data used to support the findings of this study are available from the corresponding author upon request.

\section{Conflicts of Interest}

The authors declare that they have no conflicts of interest.

\section{Acknowledgments}

It is our pleasure to acknowledge the researchers' contributions by providing their data for use in this analysis.

\section{References}

[1] A. Fenton and N. Panay, "Estrogen, menopause and joints," Climacteric, vol. 19, no. 2, pp. 107-108, 2016.

[2] A. D. Woolf and B. Pfleger, "Burden of major musculoskeletal conditions," Bulletin of the World Health Organization, vol. 81, pp. 646-656, 2003.

[3] S. F. Dworkin and L. LeResche, "Research diagnostic criteria for temporomandibular disorders: review, criteria, examinations and specifications, critique," Journal of Craniomandibular Disorders, vol. 6, no. 4, pp. 301-355, 1992.

[4] H. A. Israel, B. Diamond, F. Saed-Nejad, and A. Ratcliffe, "Osteoarthritis and synovitis as major pathoses of the temporomandibular joint: comparison of clinical diagnosis with arthroscopic morphology," Journal of Oral and Maxillofacial Surgery, vol. 56, no. 9, pp. 1023-1027, 1998.

[5] S. M. Seed, K. C. Dunican, and A. M. Lynch, "Treatment options for osteoarthritis: considerations for older adults," Hospital Practice, vol. 39, no. 1, pp. 62-73, 2011.

[6] M. Sowers, M. Hochberg, J. P. Crabbe, A. Muhich, M. Crutchfield, and S. Updike, "Association of bone mineral density and sex hormone levels with osteoarthritis of the hand and knee in premenopausal women," American Journal of Epidemiology, vol. 143, no. 1, pp. 38-47, 1996.

[7] T. D. Spector, L. A. Perry, and R. W. Jubb, "Endogenous sex steroid levels in women with generalised osteoarthritis," Clinical Rheumatology, vol. 10, no. 3, pp. 316-319, 1991.

[8] S. M. Hussain, F. M. Cicuttini, R. J. Bell et al., "Incidence of total knee and hip replacement for osteoarthritis in relation to circulating sex steroid hormone concentrations in women," Arthritis \& Rheumatology, vol. 66, no. 8, pp. 2144-2151, 2014.

[9] E. Ntoumou, M. Tzetis, M. Braoudaki et al., "Serum microRNA array analysis identifies miR-140-3p, miR-33b-3p and miR-671-3p as potential osteoarthritis biomarkers involved in metabolic processes," Clinical Epigenetics, vol. 9, no. 1, p. 127, 2017.

[10] C.-H. Chou, C.-C. Wu, I.-W. Song et al., "Genome-wide expression profiles of subchondral bone in osteoarthritis," Arthritis Research \& Therapy, vol. 15, no. 6, p. R190, 2013.

[11] K. M. Fisch, R. Gamini, O. Alvarez-Garcia et al., "Identification of transcription factors responsible for dysregulated networks in human osteoarthritis cartilage by global gene expression analysis," Osteoarthritis and Cartilage, vol. 26, no. 11, pp. 1531-1538, 2018.

[12] Y. J. Chen, W. A. Chang, L. Y. Wu, Y.-L. Hsu, C.-H. Chen, and P.-L. Kuo, "Systematic analysis of transcriptomic profile of chondrocytes in osteoarthritic knee using next-generation sequencing and bioinformatics," Journal of Clinical Medicine, vol. 7 , no. 12 , p. $535,2018$.

[13] X. Zhang, Y. Bu, B. Zhu et al., "Global transcriptome analysis to identify critical genes involved in the pathology of osteoarthritis," Bone \& Joint Research, vol. 7, no. 4, pp. 298-307, 2018.

[14] A. N. Toukap, C. Galant, I. Theate et al., "Identification of distinct gene expression profiles in the synovium of patients with systemic lupus erythematosus," Arthritis \& Rheumatism, vol. 56, no. 5, pp. 1579-1588, 2007.

[15] D. Woetzel, R. Huber, P. Kupfer et al., "Identification of rheumatoid arthritis and osteoarthritis patients by transcriptome-based rule set generation," Arthritis Research \& Therapy, vol. 16, no. 2, p. R84, 2014.

[16] R. A. Irizarry, B. Hobbs, F. Collin et al., "Exploration, normalization, and summaries of high density oligonucleotide array probe level data," Biostatistics, vol. 4, no. 2, pp. 249-264, 2003.

[17] I. Diboun, L. Wernisch, C. Orengo, and M. Koltzenburg, "Microarray analysis after RNA amplification can detect pronounced differences in gene expression using limma," BMC Genomics, vol. 7, no. 1, p. 252, 2006.

[18] A. Reiner-Benaim, "FDR control by the $\mathrm{BH}$ procedure for two-sided correlated tests with implications to gene expression data analysis," Biometrical Journal, vol. 49, no. 1, pp. 107-126, 2007.

[19] P. He, Z. Zhang, W. Liao, D. Xu, M. Fu, and Y. Kang, "Screening of gene signatures for rheumatoid arthritis and osteoarthritis based on bioinformatics analysis," Molecular Medicine Reports, vol. 14, no. 2, pp. 1587-1593, 2016.

[20] R. Ledesma-Amaro, Z. Lazar, M. Rakicka et al., "Metabolic engineering of Yarrowia lipolytica to produce chemicals and fuels from xylose," Metabolic Engineering, vol. 38, pp. 115124, 2016.

[21] D. W. Huang, B. T. Sherman, and R. A. Lempicki, "Systematic and integrative analysis of large gene lists using DAVID bioinformatics resources," Nature Protocols, vol. 4, no. 1, pp. 44-57, 2009.

[22] D. Szklarczyk, J. H. Morris, H. Cook et al., "The STRING database in 2017: quality-controlled protein-protein association 
networks, made broadly accessible," Nucleic Acids Research, vol. 45, no. D1, pp. D362-D368, 2017.

[23] D.-H. Le and Y.-K. Kwon, "NetDS: a cytoscape plugin to analyze the robustness of dynamics and feedforward/feedback loop structures of biological networks," Bioinformatics, vol. 27, no. 19, pp. 2767-2768, 2011.

[24] A. Phinyomark, S. T. Osis, B. A. Hettinga, D. Kobsar, and R. Ferber, "Gender differences in gait kinematics for patients with knee osteoarthritis," BMC Musculoskeletal Disorders, vol. 17, no. 1, p. 157, 2016.

[25] R. J. Santen, D. C. Allred, S. P. Ardoin et al., "Postmenopausal hormone therapy: an Endocrine Society scientific statement," The Journal of Clinical Endocrinology \& Metabolism, vol. 95, no. 7_supplement_1, pp. s1-s66, 2010.

[26] J. Guo, A. Sueta, K. Nakamura et al., "Genetic and environmental factors and serum hormones, and risk of estrogen receptor-positive breast cancer in pre- and postmenopausal Japanese women," Oncotarget, vol. 8, no. 39, pp. 65759-65769, 2017.

[27] Z. Wu, Z. Luan, X. Zhang et al., "Chondro-protective effects of polydatin in osteoarthritis through its effect on restoring dysregulated autophagy via modulating MAPK, and PI3K/ Akt signaling pathways," Scientific Reports, vol. 9, no. 1, p. 13906, 2019.

[28] L. Li, F. Liu, W. Huang et al., "Ricolinostat (ACY-1215) inhibits VEGF expression via PI3K/AKT pathway and promotes apoptosis in osteoarthritic osteoblasts," Biomedicine \& Pharmacotherapy, vol. 118, Article ID 109357, 2019.

[29] X. Fu, L.-F. Gong, Y.-F. Wu et al., "Urolithin A targets the PI3K/Akt/NF-kappaB pathways and prevents IL-1beta-induced inflammatory response in human osteoarthritis: in vitro and in vivo studies," Food \& Function, vol. 10, no. 9, pp. 6135-6146, 2019.

[30] Y. Fang, P. Wang, L. Xia et al., "Aberrantly hydroxymethylated differentially expressed genes and the associated protein pathways in osteoarthritis," PeerJ, vol. 7, p. e6425, 2019.

[31] Z. Li, R. Zhang, X. Yang et al., "Analysis of gene expression and methylation datasets identified ADAMTS9, FKBP5, and PFKBF3 as biomarkers for osteoarthritis," Journal of Cellular Physiology, vol. 234, no. 6, pp. 8908-8917, 2019.

[32] X. Gao, Y. Sun, and X. Li, "Identification of key gene modules and transcription factors for human osteoarthritis by weighted gene co-expression network analysis," Experimental and Therapeutic Medicine, vol. 18, pp. 2479-2490, 2019.

[33] L. Qin and F. Beier, "EGFR signaling: friend or foe for cartilage?,” JBMR Plus, vol. 3, no. 2, Article ID e10177, 2019.

[34] L. Xie, H. Xie, C. Chen, Z. Tao, C. Zhang, and L. Cai, "Inhibiting the PI3K/AKT/NF-kappaB signal pathway with nobiletin for attenuating the development of osteoarthritis: in vitro and in vivo studies," Food \& Function, vol. 10, no. 4, pp. 2161-2175, 2019.

[35] X. Liu, P. Wang, C. Zhang, and Z. Ma, "Epidermal growth factor receptor (EGFR): a rising star in the era of precision medicine of lung cancer," Oncotarget, vol. 8, no. 30, pp. 50209-50220, 2017.

[36] H. M. Asif, S. Sultana, S. Ahmed, N. Akhtar, and M. Tariq, "HER-2 positive breast cancer-a mini-review," Asian Pacific Journal of Cancer Prevention, vol. 17, no. 4, pp. 1609-1615, 2016.

[37] H. Sun, Y. Wu, Z. Pan et al., "Gefitinib for epidermal growth factor receptor activated osteoarthritis subpopulation treatment," EBioMedicine, vol. 32, pp. 223-233, 2018.

[38] T. Kitamura, M. Sekimata, S.-I. Kikuchi, and Y. Homma, "Involvement of poly(ADP-ribose) polymerase 1 in ERBB2 expression in rheumatoid synovial cells," American Journal of Physiology-Cell Physiology, vol. 289, no. 1, pp. C82-C88, 2005.

[39] X. Hu, X. Ji, M. Yang et al., "Cdc42 is essential for both articular cartilage degeneration and subchondral bone deterioration in experimental osteoarthritis," Journal of Bone and Mineral Research, vol. 33, no. 5, pp. 945-958, 2018.

[40] J. Y. Lim, K. I. Im, E. S. Lee et al., "Enhanced immunoregulation of mesenchymal stem cells by IL-10-producing type 1 regulatory T cells in collagen-induced arthritis," Scientific Reports, vol. 6, no. 1, p. 26851, 2016.

[41] C. J. Malemud, "The PI3K/Akt/PTEN/mTOR pathway: a fruitful target for inducing cell death in rheumatoid arthritis?," Future Medicinal Chemistry, vol. 7, no. 9, pp. 1137-1147, 2015.

[42] J.-F. Xue, Z.-M. Shi, J. Zou, and X.-L. Li, "Inhibition of PI3K/ AKT/mTOR signaling pathway promotes autophagy of articular chondrocytes and attenuates inflammatory response in rats with osteoarthritis," Biomedicine \& Pharmacotherapy, vol. 89, pp. 1252-1261, 2017.

[43] I. Cortes, J. Sanchez-Ruiz, S. Zuluaga et al., "p85beta phosphoinositide 3-kinase subunit regulates tumor progression," Proceedings of the National Academy of Sciences, vol. 109, no. 28, pp. 11318-11323, 2012.

[44] A. S. Marchev, P. A. Dimitrova, A. J. Burns, R. V. Kostov, A. T. Dinkova-Kostova, and M. I. Georgiev, "Oxidative stress and chronic inflammation in osteoarthritis: can NRF2 counteract these partners in crime?," Annals of the New York Academy of Sciences, vol. 1401, no. 1, pp. 114-135, 2017.

[45] M. Durand, S. V. Komarova, A. Bhargava et al., "Monocytes from patients with osteoarthritis display increased osteoclastogenesis and bone resorption: the in vitro osteoclast differentiation in arthritis study," Arthritis \& Rheumatism, vol. 65, no. 1, pp. 148-158, 2013. 


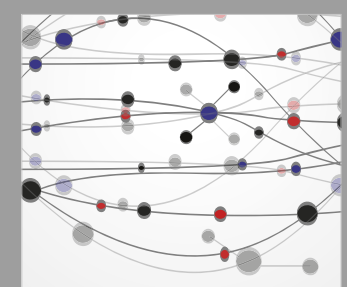

The Scientific World Journal
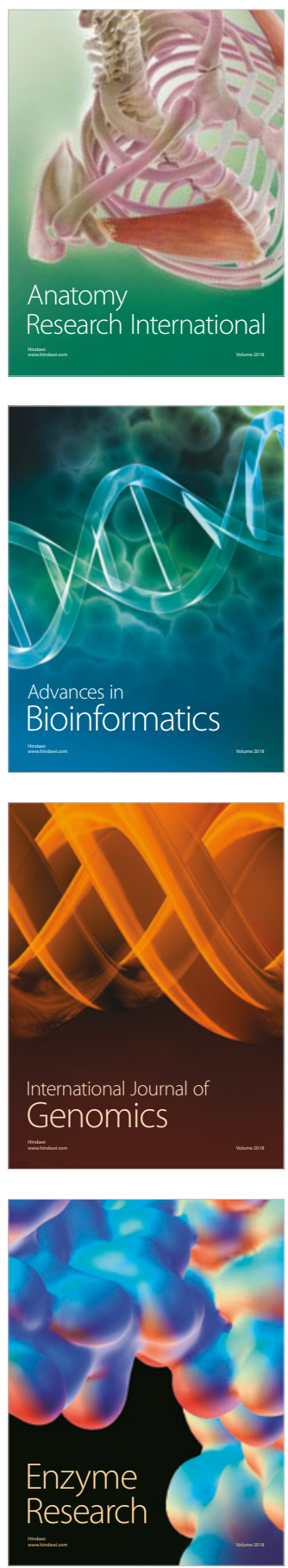
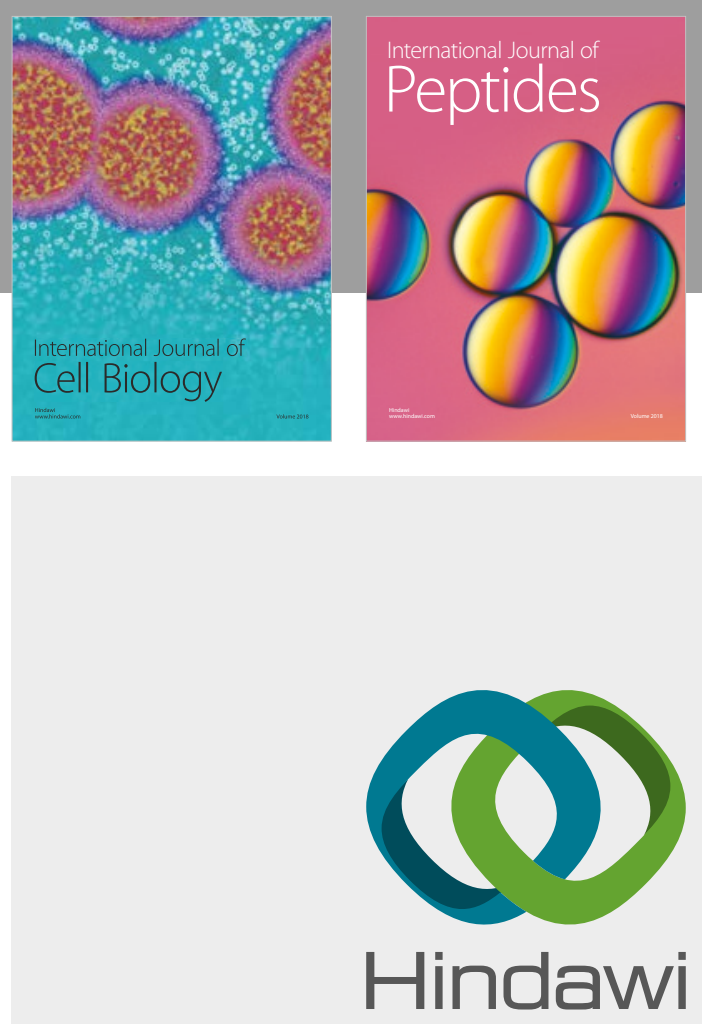

Submit your manuscripts at

www.hindawi.com
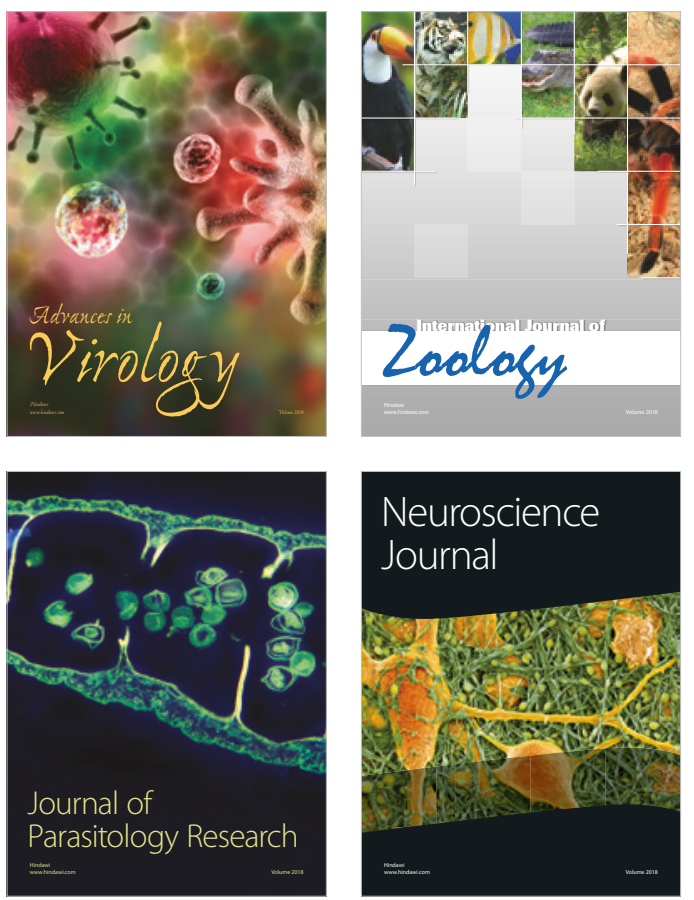
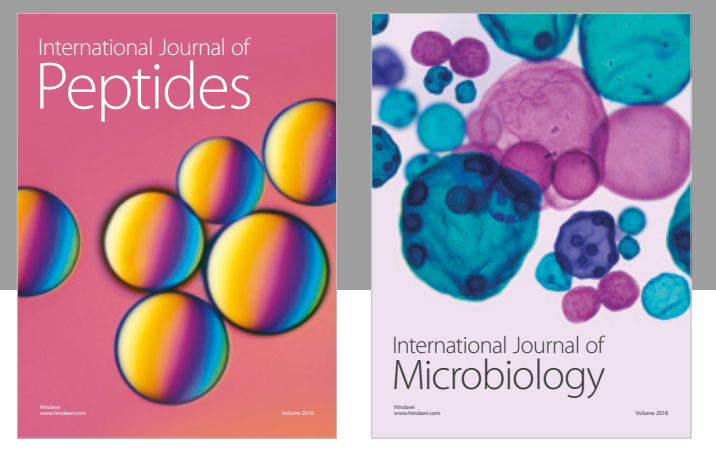

nternational Journal of Microbiology
Journal of
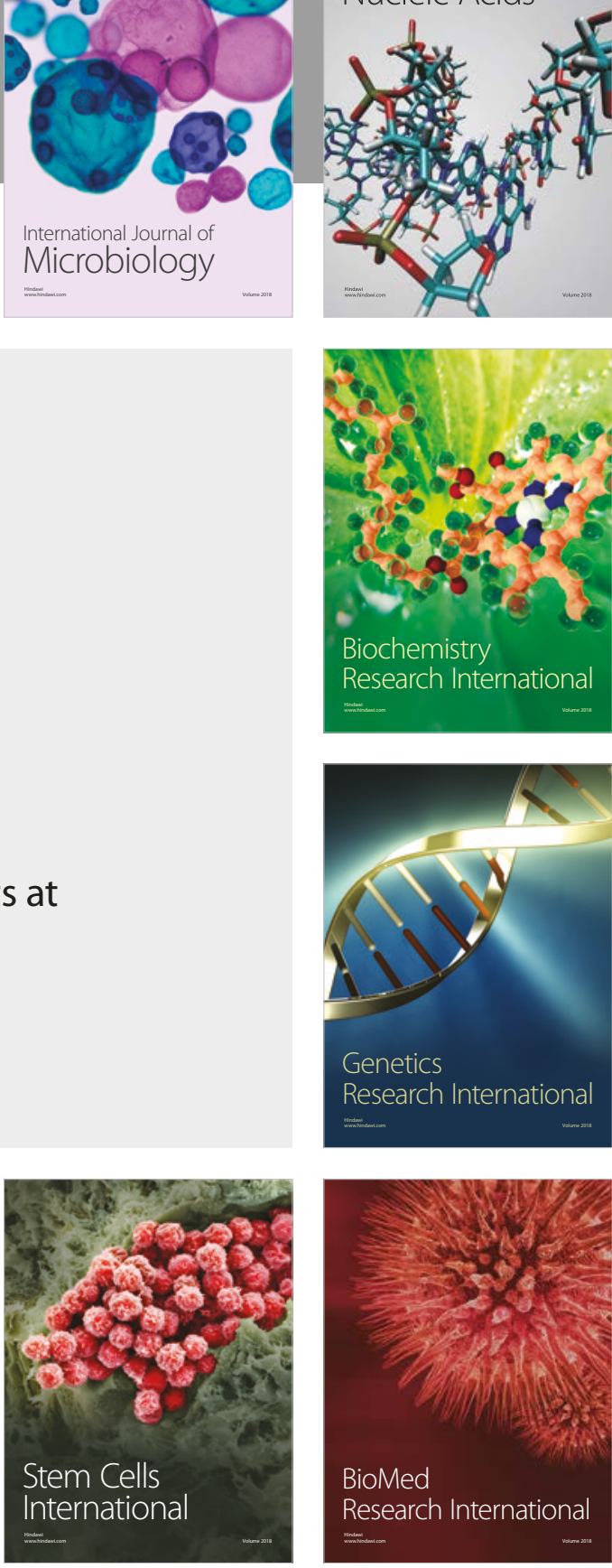
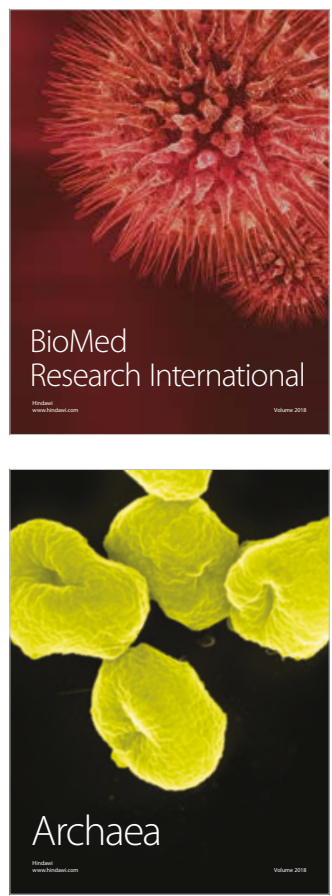